Canadian University Music Review

Revue de musique des universités canadiennes

\title{
Un manuscrit de cantiques à Montréal (XVIII ${ }^{\mathbf{e}}$ siècle)
}

\section{Élisabeth Gallat-Morin}

Volume 11, numéro 2, 1991

URI : https://id.erudit.org/iderudit/1014108ar

DOI : https://doi.org/10.7202/1014108ar

Aller au sommaire du numéro

Éditeur(s)

Canadian University Music Society / Société de musique des universités

canadiennes

ISSN

0710-0353 (imprimé)

2291-2436 (numérique)

Découvrir la revue

Citer cet article

Gallat-Morin, É. (1991). Un manuscrit de cantiques à Montréal (XVIII ${ }^{\mathrm{e}}$ siècle). Canadian University Music Review / Revue de musique des universités

canadiennes, 11(2), 68-93. https://doi.org/10.7202/1014108ar

(c) Canadian University Music Society / Société de musique des universités canadiennes, 1991
Ce document est protégé par la loi sur le droit d'auteur. L'utilisation des services d'Érudit (y compris la reproduction) est assujettie à sa politique d'utilisation que vous pouvez consulter en ligne.

https://apropos.erudit.org/fr/usagers/politique-dutilisation/ 


\title{
UN MANUSCRIT DE CANTIQUES À MONTRÉAL(XVIIIE SIÈCLE)
}

\author{
Élisabeth Gallat-Morin
}

Il y eut quantité de Demoiselles de la Ville à qui on apprenoit des Cantiques spirituels et des Motets et qui les chantoient soit au sermon, soit au salut ${ }^{1}$.

Ainsi en fut-il tout au long du Jubilé célébré à Montréal en 1729, comme le relate un Recueil des usages de la paroisse de Montréal. Mais par quels cantiques ces demoiselles rehaussaient-elles l'éclat des célébrations? Dans quel répertoire puisaient-elles?

Voici qu'un petit manuscrit d'une quarantaine de pages, surgi d'une boîte conservée à la Bibliothèque nationale du Québec à Montréal ${ }^{2}$, vient nous apporter des éléments de réponse. Ainsi, les uns après les autres, les documents du passé sortent de l'ombre, nous interpellent et nous livrent de nouvelles bribes de connaissances sur la vie de nos ancêtres.

\section{Description du manuscrit}

Le manuscrit de cantiques, de format oblong, mesurant $26,5 \mathrm{~cm} \times 19,5 \mathrm{~cm}$, comporte quarante pages de papier épais. Sur la page de garde arrière, on a copié la table; la page de garde avant manque. Le filigrane en forme d'une grosse grappe de raisin est très courant dans les papiers français du XVIII' ${ }^{e}$ siècle (cette marque indique le format du papier avant que les feuilles ne soient pliées et coupées). La contremarque, A (fleur) $\mathrm{M}$, est semblable à celui du papier de plusieurs manuscrits français de musique d'orgue de la fin du XVIIe siècle 3 . Toutefois, il est illusoire de chercher dans les filigranes une indication qui

1 Archives du Séminaire de Saint-Sulpice de Montréal, section C, rayon 2-198, Recueil des usages de la paroisse de Montréal concernant l'office divin, manuscrit, 1796, p. 131.

${ }^{2}$ L'auteur a vu ce manuscrit pour la première fois en 1988 aux Archives nationales du Québec à Montréal, qui viennent d'effectuer un important transfert de documents musicaux au profit de la Bibliothèque nationale du Québec à Montréal, Collections spéciales. Ancienne cote : ANQ-M, fonds privés, $\mathrm{P} 233 / 1$; en cours de reclassification.

${ }^{3}$ Un des cahiers du Livres d' orgue de Montréal, le manuscrit Rés. 476 conservé à la Bibliothèque nationale, Paris et le ms. 172 de la Bibliothèque municipale de Tours. 
permette une datation précise. Sur le méplat de la couverture arrière du manuscrit on peut lire, mais à l'envers, l'inscription manuscrite «À L'usage de Monsieur ». Sur le méplat de la couverture avant, également à l'envers, les mots «St Ouen » inscrits au crayon. A-t-on réutilisé la reliure d'un autre livre? Celle-ci est en veau; elle est très simple, une reliure « maison " pour ainsi dire, et très usée. À l'intérieur, elle est défaite, et les fils qui maintiennent les feuilles de papier en place se sont relâchés. De toute évidence, ce livre a été ouvert et refermé souventes fois au cours de sa carrière.

Les pages sont réglées en rouge par des portées de quatre lignes (comme pour le plain-chant). Les cinquante-trois cantiques sont copiés par la même main, mais c'est une autre main qui en a dressé la table et numéroté les pages. Pour chaque cantique, la musique est notée, avec les paroles de la première strophe inscrites sous la portée. Ce document constitue avant tout un aide-mémoire pour les airs des cantiques, car la suite des paroles n'est pas donnée.

\section{Le propriétaire du livre de cantiques}

À qui ce document appartint-il ? Il est plus que probable qu'il soit d'origine sulpicienne. En effet, dans les années 1960, plusieurs manuscrits furent transférés de l'ancienne Bibliothèque Saint-Sulpice, devenue Bibliothèque nationale du Québec, aux Archives nationales. Nombre de ces manuscrits provenaient du Séminaire de Saint-Sulpice, dont un grand antiphonaire qui avait servi au XVIII ${ }^{e}$ siècle à la paroisse Notre-Dame de Montréal ${ }^{4}$. Le manuscrit qui nous intéresse est vraisemblablement entré aux Archives nationales au même moment.

Un autre aspect du manuscrit de cantiques vient renforcer sa probable appartenance sulpicienne. Lorsque l'auteure de ces lignes entrouvrit le livre pour la première fois, l'écriture lui a semblé familière. Elle y reconnut la graphie de Jean Girard (1696-1765), ce clerc sulpicien qui fut organiste et maître d'école à Montréal pendant quarante ans. La formation complète de musicien d'église qu'il reçut à la maîtrise de la Sainte-Chapelle de Bourges fait de lui probablement le premier musicien de métier que connut Montréal. Il arriva en 1724 préparé à sa nouvelle tâche, ayant apporté avec lui plusieurs livres de musique, dont le manuscrit désormais célèbre du Livre d'orgue de Montréal, mis au jour par l'auteure en 1978. Au cours de sa carrière à Montréal, il semble avoir, en outre, couvert de sa main un nombre considérable de feuilles de plain-chant. La graphie du manuscrit de cantiques est, en effet, identique à un manuscrit de plain-chant

4 Cet antiphonaire [Cantus diversi] a encore été transféré à la Bibliothèque nationale du Québec, Montréal, Collections spéciales, avec d'autres manuscrits musicaux. Ancienne cote : ANQ-M, fonds privés, P-29. 
conservé dans les archives sulpiciennes de Montréal, dont l'écriture est comparable à celle d'un document signé par Girard (voir Gallat-Morin 1988: 104 et $64)^{5}$. Après une comparaison minutieuse de ces documents et de plusieurs autres, il est possible de poser comme hypothèse que le manuscrit de cantiques faisait partie des outils dont disposait Jean Girard dans l'exercice de son métier de musicien d'église et d'ecclésiastique enseignant, qui le plaçait au centre de la vie de la petite communauté montréalaise.

\section{L'origine des cantiques}

Est-il possible d'identifier le contenu musical du manuscrit, d'établir l'origine des cantiques? Le document ne comporte aucune indication d'auteur, à deux exceptions près. Seule existe une table des incipits littéraires des cantiques, dressée selon l'ordre dans lequel ils figurent dans le manuscrit. Afin de rendre la démarche plus facile, nous en avons établi la liste par ordre alphabétique. Tenter d'identifier les cantiques d'après les incipits musicaux eut été un travail interminable, compte tenu du vaste corpus des cantiques français et des nombreuses variantes qui existent dans les mélodies ou « timbres ».

Avant d'aller plus avant, il convient de préciser les objectifs et les limites de cette étude. La découverte de ce nouveau manuscrit nous amène à situer son contenu par rapport au répertoire analogue, à déterminer dans la mesure du possible la provenance des cantiques et l'époque à laquelle ils furent écrits ou copiés, et, enfin - c'est peut-être l'aspect le plus intéressant - à décrire les circonstances dans lesquelles le manuscrit aurait pu servir. Toutefois, devant l'énormité du corpus de cantiques français publiés aux XVII et XVIIIe siècles (dans la préface des Opuscules sacrés et lyriques de 1772, on dénombre plus de quatre-vingts livres, et la liste n'est pas exhaustive), il ne s'agit ni d'effectuer une étude approfondie du cantique français ${ }^{6}$ (les spécialistes eux-mêmes ne s'y retrouvent pas encore), ni même d'étudier le manuscrit dans le détail, l'intérêt musical de son contenu ne justifiant guère un tel effort.

Dans l'échantillonnage de livres de cantiques des XVII et $\mathrm{XVIII}$ e siècles que nous avons examinés, il a été possible de retracer les incipits littéraires, parfois avec de légères variantes, de vingt-neuf des cinquante-et-un cantiques du

5 Voir également la monographie sur Jean Girard en cours de préparation par l'auteure de cet article. Rappelons que Girard avait aussi été maitre de chant au Séminaire de Saint-Sulpice de Paris; il possédait de G.-G. Nivers (1632-1714), organiste de la paroisse Saint-Sulpice de Paris, le Premier Livre d'orgue et le Traité de la Composition de Musique.

6 J'exprime toute ma reconnaissance à Denise Launay, Jean-Yves Hameline et Jacques Cheyronnaud, dont l'expérience et les sages conseils m'ont retenue au bord du gouffre dans lequel je m'apprêtais à sauter allègrement. 
manuscrit, c'est-à-dire de plus de la moitié. Fait plus intéressant encore, la plupart des chants identifiés se retrouvent dans des livres de cantiques imprimés qui étaient présents au Québec sous le régime français, et bon nombre figurent encore dans des livres imprimés au Québec après la conquête ou importés de France ${ }^{7}$. (Voir la Table 1. Les livres ont été numérotés et les chiffres renvoient à la liste de livres de cantiques qui se trouve à la fin de cet article : les nos 1 à 4 sont du régime français, les $\mathrm{n}^{\text {os }} 5$ à 9 sont postérieurs.)

Le livre dans lequel on retrouve le plus grand nombre des cantiques du manuscrit, dix-sept au total, est le recueil publié chez la Veuve Hubault à Angers en 1735 (l'auteur est désigné par les initiales M.A.V.***P.M.), dont un exemplaire appartient à l'Hôtel-Dieu de Québec (Livre no 3 ). « Cette collection renferme tous les meilleurs cantiques \& les moins mauvais », dans l'opinion descompilateurs des Opuscules sacrés et lyriques (...) de St-Sulpice (1771) (Livre no 5). Ceux-ci sont, en effet, peu élogieux sur la qualité de la plupart des recueils qui ont précédé le leur, à l'exception de l'ouvrage d'un confrère sulpicien, M. Lagedamont, offert par J.-B. Garnier à Paris en 1750, qui renferme huit des cantiques du manuscrit (voir Livre $n^{\circ} 4$, également dans les archives de 1'Hôtel-Dieu de Québec). Ils estiment encore « fâcheux que M.l'Abbé Pellegrin ait composé tant de Cantiques; on sçait qu'il étoit en état d'en faire de bons s'il eût voulu y mettre le tems. " Cela n'a pas empêché de nombreuses éditions de ses recueils, qui connurent une grande diffusion. Les exemplaires conservés au Québec contiennent huit des cantiques du manuscrit (voir Livre $\mathrm{n}^{\mathrm{o}} 2$ ).

Plusieurs cantiques étaient de véritables airs à succès, des « tubes » dirait-on aujourd'hui. La première place est revendiquée par Reviens pécheur $\left(\mathrm{n}^{\circ} 35\right)$, qui figure dans presque tous les recueils pendant deux siècles, tout en changeant d'air selon la mode du jour. Suit de près le Noël Dans cette étable $\left(\mathrm{n}^{\circ} 8\right)$, puis Bénissez le Seigneur Suprême $\left(\mathrm{n}^{\circ} 28\right)$ et Vous qui voyez couler mes larmes ( $\left.\mathrm{n}^{\circ} 49\right)$. Trente ans après la conquête, une quinzaine des cantiques du manuscrit, dont ceux-ci, sont incorporés dans le recueil imprimé à Québec en 1795, sans airs notés, par J.-B. Boucher-Belleville, suivi en 1819 par l'aumonier des Ursulines, Jean-Denis Daulé, dont l'édition comprend la musique gravée (Livres nos 6 et 7).

L'échantillon des livres examinés est suffisant pour situer le contenu du manuscrit montréalais par rapport aux recueils de cantiques les plus couramment employés au XVIII ${ }^{\mathrm{e}}$ siècle, à la fois en France et en Nouvelle-France. S'ajoutent

7 Nous tenons la liste de la dizaine d'autres livres français dans lesquels nous avons retracé les cantiques, ainsi que de la vingtaine de livres examinés où nous n'avons trouvé aucun des cantiques du manuscrit. 


\section{Table 1}

Cantiques du manuscrit (incipits littéraires), et les livres imprimés dans lesquels on les trouve

(Les livres sont numérotés ; les chiffres renvoient à la Table 4)

Livres nos. :

Cantiques:

2 Ce bas séjour

3 Désert de ton profond silence

5 Craignez un avenir fâcheux

6 Avancez mon trépas

7 Grand Dieu que de merveilles

8 Dans cette étable

10 Sous le firmament

11 Je vis mais c'est en Dieu

13 Un Dieu vient se faire entendre

14 D'un amour extrême

18 Grand Dieu j'en n'en puis plus

19 Avancez mon trépas

23 De ton heureux retour

24 Chrétiens voulez-vous être heureux

28 Bénissez le Seigneur Suprême

30 Afin d'être docile et sage

32 Seigneur pour être à vous

33 Adorons tous dans ce profond mystère

34 Adore un Dieu qui seul est adorable

35 Reviens pécheur

36 Suivons Jésus, c'est lui qui nous mène

38 Pécheur obstiné

40 Cher enfant qui vient de naître

44 Auguste et Divine Marie

460 mon bon Jésus

48 En secret le Seigneur

49 Vous qui voyez couler mes larmes

50 Dieu de bonté, c'est ton amour

51 Quelle réjouissance

\section{$\begin{array}{lllllllll}1 & 2 & 3 & 4 & 5 & 6 & 7 & 8 & 9\end{array}$}

$\begin{array}{cccccccc} & 2 & & & 2 & & 2 & 2 \\ 3 & & & & 3 & 3 & 3 & \\ & 5 & 5 & & & & & \\ 6 & & & 6 & 6 & & \\ 7 & 7 & & 7 & 7 & & \\ & & 8 & 8 & 8 & 8 & 8 & 8 \\ & & 8 & 8 & & & \\ 10 & & & 10 & 10 & & \\ 11 & 11 & 11 & 11 & & & \end{array}$

13

14

18

19

19

24

28*

30

$\begin{array}{llll}28 & 28 & 28 & 28\end{array}$

3030

32

33

$\begin{array}{lll}34 & 34 \quad 34\end{array}$

$\begin{array}{lllllll}35 & 35 & 35 & 35 & 35 & 35 & 35\end{array}$

36

$38 \quad 38$

40

$40 \quad 40 \quad 40$

4444

46

48

$49 \quad 49$

$49 \quad 4949$

50

50

*air seulement 
à ces livres imprimés les manuscrits conservés dans diverses communautés religieuses, Ursulines, Jésuites, Sulpiciens, qui puisent au même répertoire. C'est dire que le recours à ces cantiques, comme moyen de récréation ou comme outil pédagogique, était bel et bien entré dans les mœurs de ce côté-ci de l'Atlantique. On peut constater, en outre, que cette pratique ne s'est pas perdue après le changement de régime.

\section{Destination et fonctions}

À qui étaient destinés ces cantiques? Quelle était leur fonction? Ayant retracé plus de la moitié des cantiques du manuscrit dans des livres des XVII et XVIIIe siècles, publiés en France et conservés au Québec, il convient de nous interroger maintenant sur leur utilisation, en premier lieu dans leur pays d'origine, puis une fois transportés en Nouvelle-France.

La première constatation que nous pouvons faire est que les cantiques français de cette époque sont loin de former un corpus homogène. Ils ne visent pas, non plus, une clientèle unique. Comme l'explique le Père Jean-Yves Hameline,

les auteurs, compilateurs, éditeurs de cantiques s'adressent à deux clientèles tout à fait différentes. D'un côté, le monde des missions, des exercices paroissiaux, des catéchismes. De l'autre, celui des dévôts, des cercles pieux, des « jeunes personnes » dont la pratique musicale est l'ornement nécessaire de certaines églises et chapelles de religieux.

(Hameline $1981: 252$ ).

On peut distinguer, en effet, d'une part une « pratique vocale individuelle et intime à usage domestique » et, d'autre part, une « pratique vocale collective (...) missions, catéchismes, maisons d'éducation » (Cheyronnaud $1988: 243$ ). En Nouvelle-France, on trouvera trace des deux fonctions du cantique, l'une d'ordre pédagogique, l'autre de nature récréative.

\section{La fonction pédagogique}

Comme soutien pédagogique, le cantique vise « l'endoctrinement, au sens élémentaire du terme: inculquer la doctrine » (Cheyronnaud 1988: 233). Un peu à la manière des vitraux des cathédrales, le cantique met les articles de foi et de morale à la portée des gens simples.

Il est à souligner que le cantique n'a jamais fait partie intégrante de la liturgie, dont la partie chantée se déroulait en latin et en plain-chant. Mais les fidèles du XVIII ${ }^{2}$ siècle sanctifiaient les dimanches et fêtes par une fréquentation de l'église qui dépassait la simple présence à la Messe. De nombreux autres exercices spirituels leur étaient proposés : vêpres, saluts, catéchismes ou 
instructions du dimanche, sans compter les événements moins courants qu'étaient les missions et les jubilés. C'est dans le cadre de ces manifestations « auxiliaires » que le cantique trouve son utilité, comme dans le Jubilé tenu à Montréal en 1729. À cet égard, il est bien spécifié que les « demoiselles de la ville » chantaient les cantiques « soit au sermon » (qui pouvait avoir lieu après la Messe) « soit au salut », et non dans le cadre de la liturgie proprement dite.

La tenue de Missions dans les paroisses avait pour objet de raviver la foi des fidèles, et le titre de nombreux livres de cantiques confirme leur utilisation dans ce cadre. Parmi les plus engagés dans cette forme d'apostolat, on compte le Père Louis-M. Grignon de Montfort (1673-1716) et ses disciples, dont les collections de cantiques renferment des chants des plus connus à l'époque. On en trouve au moins cinq dans le manuscrit montréalais: Avancez mon trépas $\left(\mathrm{n}^{\circ} 6\right)$, Chrétiens voulez-vous être heureux ( $\left.\mathrm{n}^{\circ} 24\right)$, Adorons tous dans ce profond mystère ( $\left.\mathrm{n}^{\circ} 33\right)$, Reviens pécheur ( $\left.\mathrm{n}^{\circ} 35\right)$ et $O$ mon bon Jésus $\left(\mathrm{n}^{\circ} 46\right)$ (Fradet 1929). Un des seuls chants du manuscrit, dont la provenance est indiquée, servait d'ouverture aux missions. Il s'agit du Cantique de $\mathrm{Mr}$ de Bridaine - Un Dieu vient se faire entendre $\left(\mathrm{n}^{\circ} 13\right)$ ([Bridaine] 1734), dont les paroles exhortent les paroissiens :

Accourez peuples fideles

Venez a La mission

Le Seigneur qui vous appelle

Veut votre conversion.

Les « catéchismes » n'avaient pas lieu dans le seul cadre scolaire; il y avait dans les paroisses un effort " d'éducation permanente " à l'égard d'une population majoritairement ignorante et peu éduquée. Un petit Coutumier manuscrit de 1728, conservé dans les Archives du Séminaire de Saint-Sulpice de Montréal, précise le calendrier des catéchismes dans la paroisse; dans certains jours de grande célébration, lorsque la Messe risque notamment de trop se prolonger, il n'y a point de catéchisme.

Il est vrai que le principal manuel scolaire utilisé à Montréal au XVIIIe siècle, L'Escole paroissiale, dont un exemplaire de 1654 se trouve à la Bibliothèque nationale du Québec à Montréal, ne mentionne pas le cantique spécifiquement, bien qu'on y fasse allusion à l'apprentissage du plain-chant. Néanmoins, d'autres ouvrages scolaires font état du cantique, dont ceux du renommé pédagogue Jean-Baptiste de La Salle, fondateur des Frères des écoles chrétiennes. Plusieurs de ses livres étaient connus dans la colonie (un certain nombre sont encore conservés dans le Fonds ancien de la Bibliothèque du Séminaire de Québec) et n'oublions pas que les écoles lasalliennes de la paroisse Saint-Sulpice 
de Paris ont formé un maître d'école pour Montréal, Antoine Forget, au début du XVIII ${ }^{e}$ siècle. Il n'est pas impossible non plus que Jean Girard ait effectué un stage dans ces mêmes petites écoles, avant de venir en Nouvelle-France. Toujours est-il qu'en plus d'avoir compilé un livre de cantiques, La Salle, dans sa Conduite des écoles, mentionne leur utilisation à plusieurs reprises. À la sortie des classes, notamment, les plus grands chantent des cantiques pendant que les petits sortent et, lors du catéchisme du dimanche, on conclut par « quelques versets de cantiques (...) ensuite on conduira les Ecoliers à Vespres » (La Salle $1720: 10,108)$. Notre manuscrit comprend quatre des cantiques qui figurent dans diverses publications lasalliennes, mais pas exclusivement : $O$ mon bon Jésus ( $\left.n^{\circ} 46\right)$, En secret le Seigneur $\left(\mathrm{n}^{\circ} 48\right)$, Vous qui voyez couler mes larmes $\left(n^{\circ} 49\right)$ et Afin d'être docile et sage $\left(n^{\circ} 30\right)$, tiré des Cantiques qui se doivent chanter avant le Catéchisme ([La Salle] 1705), avec son texte d'une fraîcheur enfantine :

Afin d'être docile et sage Seigneur

Donnez moy vostre esprit

Pour apprendre selon mon age

Les verités de Jesus Christ.

Il y a un autre cantique du manuscrit, que nous n'avons pas réussi à retracer, qui s'adresse manifestement aux jeunes. Il s'agit du $n^{\circ} 52$, Aimons Dieu dans la jeunesse.

Il incombait tout naturellement au maître d'école d'apprendre à ses élèves à chanter des cantiques, comme en témoignent nombre de contrats d'engagement. Le document de fondation d'une école de charité à Bourges, ville d'origine de Jean Girard, précise que « le maitre instruira lesd[its] ecoliers de la Religion Catholique apostolique et Romaine a lire Ecrire et de Larithmetique et a chanter des cantiques ${ }^{8}$, jusqu'au Règlement de la Congrégation des externes de SainteFamille, Ile d'Orléans, établissement fondé en 1685 par la Congrégation NotreDame, dont les jeunes filles « tous les jours (...) s'entretiendront de bons discours ou chanteront des cantiques spirituels » (Gosselin 1911 : 474).

\section{En guise de récréation}

Le célèbre Abbé Pellegrin, dont les livres ont survécu au Québec en plusieurs exemplaires, dédie son Recueil de cantiques spirituels de 1728 à l'Abbesse de Saint-Rémy, avec l'espoir que son exemple et son prestige incitent ses propres

8 Bibliothèque municipale de Bourges, GG 147 (22), Fondation de l'école de la charité, 3 avril 1719. 
religieuses, ainsi que celles d'autres communautés « à faire retentir les voutes de leurs Monasteres des loüanges du Seigneur qui sont contenües dans ce Recueil (...) » (Livre $n^{\circ} 2 b$ ). En effet, dans les couvents, ces cantiques servaient de « récréation pour jeunes filles qui connaissaient les airs du monde » avant leur entrée en religion (Cheyronnaud $1988: 243$ ). Cette pratique se poursuivit dans le Nouveau Monde. Ainsi, on peut lire l'inscription suivante dans un livre de Cantiques Spirituels appartenant à l'Hôtel-Dieu de Québec : « Ce livre est donné a linfirmerie par ma Sr Ste Catherine avec la permission de la Ste obeissance » $\left(\operatorname{Livre~}^{\circ} 1\right)$. Le manuscrit de cantiques qui fait l'objet de la présente étude devait servir à des fins semblables au Séminaire de Montréal, comme nous le verrons.

Les cantiques publiés par l'Abbé Pellegrin s'adressaient non seulement aux religieuses, mais aussi aux " personnes véritablement pieuses ». En effet, la plupart des recueils qui ont vu le jour aux XVII ${ }^{\mathrm{e}}$ et XVIII ${ }^{\mathrm{e}}$ siècles étaient " surtout destinés à la société cultivée pour d'éventuelles récréations musicales » (Hameline $1981: 251$ ), dans un effort d'évacuer des esprits les paroles mondaines et frivoles, quand elles n'étaient pas franchement licencieuses, des chansons à la mode. Le Père Surin, Jésuite,

a voulu donner une matiere plus sainte qui édifie et instruise les ames en divertissant les esprits, et les divertissent en les instruisant; afin que toute la vie du Chrétien se passe utilement dans la pratique des vertus; et de sanctifier jusques à nos plus petites recreations (Livre $n^{\circ} 1$ ).

Les livres de cantiques spirituels proposent des paroles édifiantes sur des airs connus qui circulaient dans tous les milieux.

Leur sens ôte à leurs sons un souvenir pervers,

Et sçait des chants impurs purifier les airs,

proclame la charmante Préface en vers d'un petit livre marqué « a lusage de $\mathrm{Sr}$ St. Gabriel de lhotel Dieu » [de Québec] (Livre ${ }^{\circ} 3$ ). On peut penser qu'en Nouvelle-France, comme dans la mère-patrie, des livres de cantiques spirituels se trouvaient non seulement dans les couvents, mais aussi dans les bibliothèques des particuliers, même si d'après les inventaires après décès, ce sont les livres de chansons à boire qui semblent l'emporter.

\section{Le support musical}

Dans le cantique, le « timbre » ou air sert essentiellement de véhicule aux paroles, qui en constituent l'élément le plus important. Si pour certains cantiques la musique a été composée spécialement, dans la plupart des cas on a emprunté un 
air déjà connu du public. Plusieurs recueils de cantiques, en effet, comme les Cantiques Spirituels publiés chez la Veuve Hubault à Angers en 1737 (Livre $\mathrm{n}^{\circ} 3$ ), ne comprennent aucune notation musicale; au début de chaque cantique on trouve l'indication "Sur l'air de ", suivi du nom d'un chant connu. Pour faciliter les choses, certains auteurs de cantiques, dont l'Abbé Pellegrin, ont réemployé pour de nombreux cantiques différents un timbre qui était déjà inscrit dans la mémoire du lecteur.

Les titres des recueils précisent que les cantiques sont « mis sur les Airs les plus beaux et les plus connus » (Livre ${ }^{\circ} 3$ ), ou encore « sur les grands airs, $\&$ les airs de Musique instrumentale » $\left(\operatorname{Livre}^{\circ}{ }^{\circ}\right)$. Dans quel répertoire puisait-on ces airs? Quels sont les timbres les plus fréquemment employés? Est-il possible d'en retracer dans le manuscrit montréalais ?

Les tables qu'on trouve dans les recueils de cantiques donnent souvent une liste des airs employés et les présentent sous deux rubriques principales : « airs d'opéras » et « vaudevilles ».

Les airs d'opéras les plus connus semblent avoir été ceux de Jean-Baptiste Lully, dont on dit que les airs étaient fredonnés par le peuple. Toutefois, on indique dans une préface que ces parodies spirituelles « sont pour ceux qui, ayant plus de goût pour les chants, sçavent retenir les airs d'Opera, \& autres airs difficiles » (Livre $\mathrm{n}^{\circ} 3$ ). On emploie aussi dans les recueils les airs d'opéras de Campra, ainsi que les airs de pièces vocales et instrumentales de Couperin, Clérambault et Rameau, entre autres. Les cantiques faits sur cette musique « savante » convenaient sans doute davantage aux personnes qui avaient étudié la musique.

Le genre populaire par excellence était le vaudeville (ou voix de ville), qui s'est répandu vers la fin du XVII ${ }^{e}$ siècle. Transmis oralement, ces chants connurent un tel succès que Ballard publia en 1717 des chansons « sur des airs répandus dans le public », La Clef des chansonniers . Avec les vaudevilles, on disposait d'un « ensemble de supports mélodiques véhiculaires, dans une société où il est incontestable que le vecteur oral-musical constitu[ait] un des éléments majeurs de communication culturelle (...), [le cantique étant ainsi] doté d'un support et d'une forme qui puissent lui donner les chances d'une pratique vraiment étendue, au moins virtuellement, à la population dans son ensemble » (Hameline 1981 : 252). Une vaste collection de ces vaudevilles fut publiée par Le Sage et D'Orneval dans le Théâtre de la Foire entre 1721 et 1737 et un grand nombre d'entre eux survécurent jusqu'au XIX ${ }^{\mathrm{e}}$ siècle, en compagnie d'airs plus récents, dans la Clé du Caveau (première édition 1811), recueil qui n'était pas inconnu au Québec. D'ailleurs, le folkloriste et ethnologue Conrad Laforte a retracé jusque dans les sources que nous venons de mentionner nombre de timbres des 
chants que lui et ses collègues des Archives de folklore de l'Université Laval ont recueilli au Québec (Laforte 1983).

Examinons maintenant de plus près quelques cantiques du manuscrit faits soit surdes airs d'opéras, soit sur des airs de vaudevilles, en nous arrêtant tout d'abord au mode de notation musicale employé dans notre document.

\section{La notation musicale dans le manuscrit}

Dans la plupart des recueils imprimés qui contiennent les airs notés des cantiques, la musique est notée sur cinq lignes. Il est frappant de constater que dans le manuscrit, les cantiques sont notés, à la manière du plain-chant, sur quatre lignes ; les valeurs de notes également sont représentées comme dans le plainchant. En parcourant les divers exemples musicaux, on constatera qu'il n'y a pas de mesures comme dans la « musique ». Il y a bien des barres de division, mais celles-ci n'ont rien à voir avec la métrique ; elles semblent plutôt constituer une aide visuelle à la lecture des groupes de mots. À noter également les ornements (tremblements) ainsi que les petits phrasés sur des paires ou des groupes de notes rapides.

Des exemples existent de recueils de cantiques imprimés en plain-chant. Les Opuscules sacrés et lyriques (Livre $\mathrm{n}^{\circ}$ 5), mentionnent les « Cantiques notés en plein-chant » du recueil publié chez Robert Ballard en 1660, Cantiques sur les prieres du Chrétien. Plus près de nous, l'Abbé Daulé, dans son Nouveau recueil de cantiques àl' usage du diocese de Quebec (1819) «a ajouté une courte méthode pour apprendre à les mettre en plein chant $»\left(\right.$ Livre $\left.n^{\circ} 7\right)$. Cette méthode donne notamment les équivalences de valeurs de notes entre la « musique » et le plainchant (exemple 1). Il y avait donc des personnes, sans doute principalement les chantres d'église, qui avaient l'habitude de solfier le plain-chant sur quatre lignes, mais qui ne lisaient pas la musique sur cinq lignes avec la clef de sol, comme le pouvaient les personnes qui avaient reçu une éducation musicale plus poussée.

\section{Les cantiques du manuscrit}

Comme dans les recueils publiés, on trouve dans le manuscrit plusieurs types de cantiques, des plus simples aux plus élaborés. Certains sont faits sur des airs d'opéras, d'autres adoptent comme support les airs de vaudevilles, d'autres encore ont peut-être fait l'objet de musiques originales.

Il n'a pas toujours été facile d'identifier les airs qui ont servi de support aux cantiques du manuscrit. Les paroles d'un cantique donné ont pu être retrouvées dans un recueil imprimé sans airs notés, avec la seule mention « sur l'air de ». À 


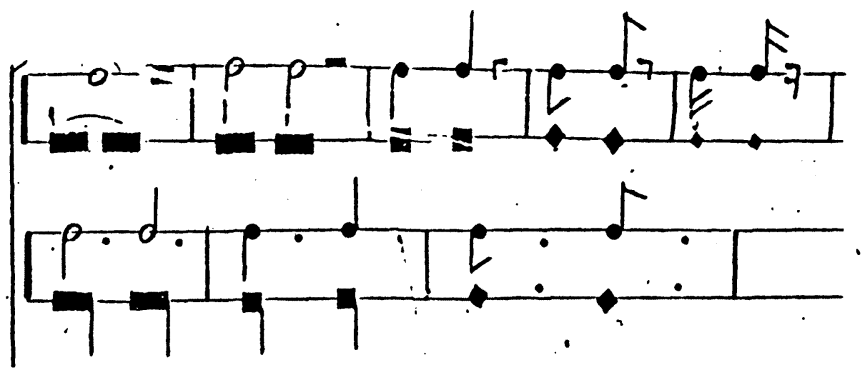

Example 1: DAULÉ (1819), Méthode pour mettre les cantiques en plain-chant

Table 2

Cantiques du manuscrit (incipits littéraires) qui n'ont pas été retracés

1 Jusqu'à vous j'élève mon âme

4 Loin de mon Dieu

9 Que je suis affligé

12 Regnez mon unique vainqueur

$15 \mathrm{~J}$ 'ay vecu dans le crime

16 L'innoncence est une rose

17 Un cœur qui vit dans l'innocence

20 Que je suis affligé

21 Sans plus attendre

22 Monde en vain par tes caresses

25 Monde trompeur en vain tu nous rappelle

$26 \mathrm{Ca}$ bergers plus de tristesses

27 C'en est fait je reviens a vous

29 Sans te flatter d'une grace nouvelle

31 Ah qu'un Dieu enfant parait charmant

37 Qu'on cherche qu'on aime

39 Charitable Marie

41 Divin esprit venez dans nos ames

42 Pourquoy donner nos jours

$43 \mathrm{La}$ sagesse n'est point severe

$45 \mathrm{Ne}$ vous laissez point seduire

47 Il faut pecheur

52 Aimons Dieu dans la jeunesse

53 Vous me cherchez mon Sauveur 
Table 3

Quelques timbres ou airs des cantiques

\section{Airs d'opéra}

D’André Campra, Hesione :

Aimable vainqueur

De Jean-Baptiste Lully, Atys :

Quand le péril est agréable

De Lully, Amadis :

Suivons l'amour, c'est lui

qui nous mène

De Campra, L'Europe galante :

Vous brillez seule en ces retraites
39 Pécheur obstiné

28 Bénissez le Seigneur Suprême

36 Suivons Jésus, c'est lui qui nous mène

49 Vous qui voyez couler mes larmes

\section{Vaudevilles}

Guai lan la lan lire

Ne m'entendez-vous pas

Petits oiseaux rassurez-vous

Prens ma Philis, prens un verre
51 Quelle réjouissance

19 Avancez mon trépas

50 Dieu de bonté, c'est ton amour

40 Cher enfant qui vient de naître

partir du nom du timbre, il a fallu le repérer dans un autre recueil qui comportait les airs notés, pour ensuite comparer avec la musique du manuscrit en vue de déterminer si l'air était le même, ce qui n'est pas toujours le cas. En effet, les mêmes paroles peuvent prendre comme support plusieurs airs différents et, inversement, le même air peut accompagner des paroles différentes.

\section{Sur des airs d'opéra}

Nous avons repéré dans le manuscrit quatre airs d'opéras qui ont servi de support aux cantiques, deux de Lully et deux de Campra (voir Table 3).

En adaptant l'air du prologue de l'opéra Amadis de Lully, Suivons l' amour, c'est lui qui nous mène , on a tout simplement remplacé le mot " amour » par « Jésus », pour en faire le cantique Suivons Jesus, c'est lui qui nous mène ( $\left.\mathrm{n}^{\circ} 36\right)$. Il n'y a évidemment aucune difficulté à adapter ces paroles à l'air de l'opéra ; la version du manuscrit est faite sur exactement le même air que celui qui est noté par Pellegrin (Livre $\mathrm{n}^{\circ} 2$ ).

Si on retrouve ce cantique dans plusieurs recueils, l'air de Lully qui a eu la carrière la plus longue est l'air de Sangaride, tiré de l'Acte I, scène III, de l'opéra 
Atys, Quand le péril est agréable. Cet air sert de support à de nombreux cantiques de Pellegrin, mais on le trouve dans notre manuscrit sous sa forme la plus répandue : Bénissez le Seigneur Suprême ( $\left.\mathrm{n}^{\circ} 28\right)$. Ce cantique devint tellement connu qu'après quelque temps, l'air était toujours désigné par le titre Bénissez et non plus d'après l'incipit littéraire de l'air de Lully Quand le péril. C'est en effet sous les paroles et l'air de Bénissez le Seigneur qu'on trouve ce cantique dans plusieurs livres français présents au Québec à la fin du XVIII e siècle et au début du XIX ${ }^{\mathrm{e}}$ siècle, ainsi que dans les livres de cantiques imprimés à Québec (voir Livres nos 5, 6, 7 et 9). Comme le fait observer Jacques Cheyronnaud,

paradoxalement, ce support mélodique - comme d'autres - passera et se maintiendra dans la modernité à travers les recueils de cantiques, fonctionnant ainsi depuis près de deux siècles dans le champ de l'hymnodie catholique d'usage, tandis que l'opéra de Lully sera relégué aux oubliettes du passé, promu, dans cette modernité, aux « redécouvertes » de la musicologie et à la restitution (Cheyronnaud $1988: 239)^{9}$.

Nous avons pu comparer le cantique du manuscrit Bénissez le Seigneur Suprême avec l'air original de Lully, dans la version gravée par H. de Baussen en 1709, trente-trois ans après la création de l'opéra. Ce livre appartenait à François Vachon de Belmont, supérieur des Sulpiciens, décédé à Montréal en 1732.10 En tête du prologue et de chaque acte est placée une gravure représentant les personnages de la pièce. Comme il s'agit parfois de déesses vêtues fort légèrement, on a pudiquement recouvert d'encre les décolletés et même les visages des personnages féminins ; n'oublions pas que nous sommes dans un séminaire d'hommes au XVIII' siècle. En outre, des paroles considérées trop frivoles sont remplacées ou carrément biffées. Dans la marge, en face de deux airs, Quand le péril est agréable et D'une constance agréable, on a inscrit au crayon le mot «Pelegrin ». C'est une référence directe aux recueils de l'Abbé Pellegrin, qui s'est servi de ces timbres pour plusieurs de ses cantiques. Comme le supérieur de Paris encourageait François de Belmont à jouer de son luth pour la consolation de ses frères, on peut très bien l'imaginer au Séminaire de Montréal accompagnant les airs de Lully mis en cantiques (le livre gravé comprend la basse chiffrée).

9 Jacques Cheyronnaud a dressé un tableau des variantes mélodiques de douze versions de ce cantique datant de 1727 à 1934 (Cheyronnaud $1988:$ 240-241).

10 L'opéra Atys est conservé à la Bibliothèque nationale du Québec, Montréal, Collections spéciales, avec cinq autres opéras de Lully ayant appartenu à Vachon de Belmont; celui-ci, on le sait, jouait du luth et possédait en France un clavecin et un orgue, qui est probablement l'instrument installé au début du XVIIIe siècle dans l'église paroissiale de Montréal. 
En composant le cantique Bénissez le Seigneur Suprême, on a remplacé les paroles écrites par Philippe Quinault pour l'air de Sangaride :

Quand le péril est agréable

Le moyen de s'en allarmer;

Est-ce un grand mal de trop aymer,

Ce que l'on trouve aymable!

par un texte tout à fait différent :

Benissez le Seigneur Supreme

Petits oiseaux dans nos forets

Dites sous ces ombrages frais

Dieu merite qu'on l'aime.

Si l'on compare la notation musicale du manuscrit avec la version gravée (exemples $2 \mathrm{a} \& \mathrm{~b}$ ), on constatera que de très légères adaptations ont dû être faites dans la mélodie du cantique pour tenir compte des nouvelles paroles. Le soupir de la mesure 5 du texte de Lully a été remplacé par une note, pour accommoder les deux syllabes du mot " petits », tandis que dans la mesure suivante, la seconde syllabe de « oiseaux » est prolongée sur les trois temps que dure la mesure, supprimant la seconde note de l'air original. Dans la première mesure du cantique, les trois première notes sont égales, tandis que l'air de Lully ouvre sur un motif de noire-noire pointée-croche ; toutefois, lorsqu'il est repris à la mesure 3 , on trouve le même motif rythmique dans le cantique. (L'équivalence des valeurs de notes entre la notation en « musique » et en plain-chant est fournie par l'exemple 1.) Il est à noter, en outre, que tous les ornements de l'air de Lully sont conservés dans le cantique. On voit dans l'exemple 2c comment a évolué l'air de Lully, qui est présenté sous le titre Bénissez le Seigneur dans le recueil imprimé à Québec par l'Abbé Daulé en 1819 (Livre n 7); dans le même ton que l'original, en 6/8 au lieu de 3, la musique suit d'assez près la mélodie originale.

Un autre compositeur bien connu en Nouvelle-France est André Campra, dont les livres de motets existent encore à Québec en une douzaine d'exemplaires, sans compter les copies manuscrites. Le manuscrit de cantiques comprend deux chants faits sur des airs d'opéras de ce compositeur. Pécheur obstiné $\left(\mathrm{n}^{\circ} 38\right)$ est fait sur un air de l'opéra Hésione, Aimable vainqueur. La musique notée dans le manuscrit suit de près l'air donné par Pellegrin (Livre $n^{0} 2$ ) ; les paroles du cantique sont remplies du courroux de Dieu qui s'apprête à lancer ses foudres sur la tête du pécheur non repenti. L'air Vous brillez seule en ces retraits, tiré de l'opéra L'Europe galante, sert de support au cantique très répandu Vous qui voyez couler mes larmes $\left(\mathrm{n}^{\circ} 49\right)$, dont l'air figure aussi dans les recueils de Pellegrin. Nous n'avons pu comparer la musique avec les originaux de Campra, 
mais dans le cas de l'adaptation de l'air de Lully, il n'y avait pas une grande différence entre l'air original et le cantique.

Sur des airs de vaudevilles

Plusieurs des airs des cantiques du manuscrit font partie du vaste corpus de vaudevilles transmis par les divers recueils que nous avons évoqués (voir Table 3).

Ne m'entendez-vous pas fait partie de ces airs connus que Conrad Laforte a retracés dans le Théâtre de la Foire et La CléduCaveau. Nous avons pu constater que l'air du cantique ${ }^{\circ} 19$, Avancez mon trépas, suivait le même air, jusque dans les ornements. Le recueil de la Veuve Hubault (Livre $n^{\circ} 3$ ) indique le même timbre (non noté) pour des paroles qui comportent de légères variantes. Dans le manuscrit, il existe une seconde version d'Avancez mon trépas $\left(\mathrm{n}^{\circ} 6\right)$, qui emprunte un autre air. Les paroles de ce cantique sont très représentatives de la spiritualité véhiculée par le manuscrit, qui repose sur le plus grand détachement possible du monde, rempli d'embûches pour le salut des âmes. Cette sévérité dont on a perdu l'habitude est tempérée, toutefois, par l'amour de Dieu pour les hommes et l'amour que les fidèles doivent à leur tour ressentir pour Lui.

Avancez mon trepas

Jesus ma douce vie

Car mon ame s'ennuye

De rester icy bas

Ne vous y voyant pas $\left(n^{\circ} 6\right)$.

Il ne faut pas s'étonner de voir les vaudevilles accouplés à un autre genre tout aussi populaire, les noëls, dont six se retrouvent dans le manuscrit. Quelle réjouissance $\left(\mathrm{n}^{\circ} 51\right)$ adopte l'air Au gai lan la, lan lire, qui sont les dernières paroles du refrain dans la version de l'Abbé Pellegrin ; à la place, les versets du manuscrit se terminent par deux alleluya ornés.

Il y a un autre noël qu'on trouve dans plusieurs recueils français du XVIII siècle et qui est encore chanté au Québec pendant la période des fêtes de fin d'année. Fait sur le vaudeville Prens ma Philis, prens un verre, inclus dans La clé du Caveau, le noël Cher enfant qui vient de naître $\left(\mathrm{n}^{\circ} 40\right)$ figure dans tous les recueils publiés au Québec par Boucher-Belleville, Daulé, Neilsen, et jusque dans la collection d'Ernest Gagnon (1909), qui s'était donné pour mission de remettre le patrimoine musical québécois à l'honneur. Après avoir comparé la musique de plusieurs versions (l'exemple 3 en donne la première ligne), nous avons pu constater à quel point ce noël traditionnel était resté intact à travers les années. 

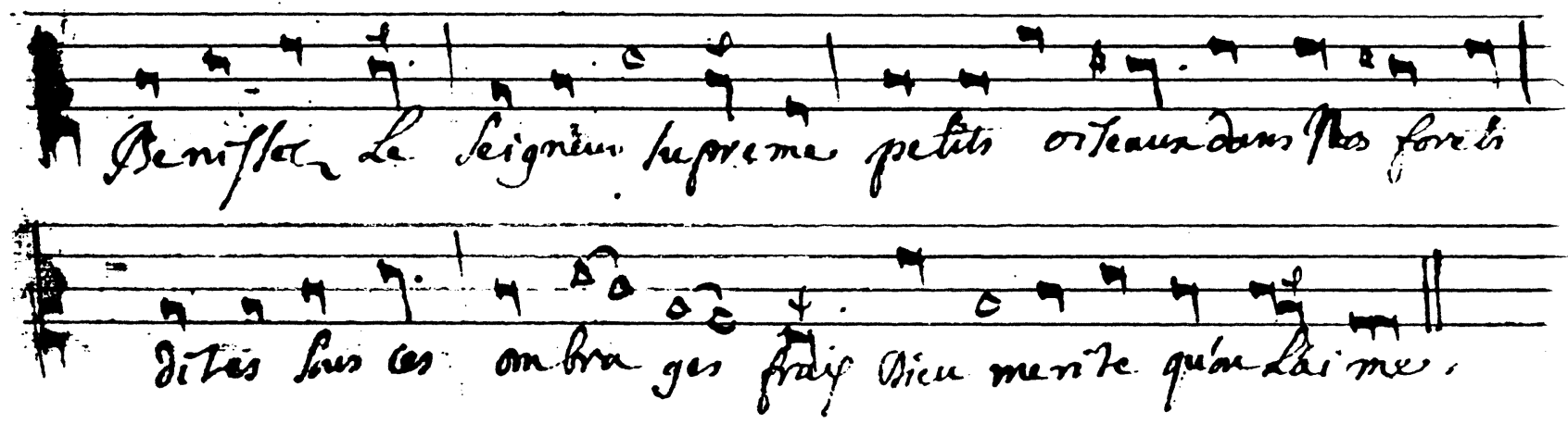

N.B. Afin de rendre la reproduction lisible, il a été nécessaire de retracer les lignes de la portée.

Example 2a : Cantique no 28, Benissez le Seigneur Supreme 

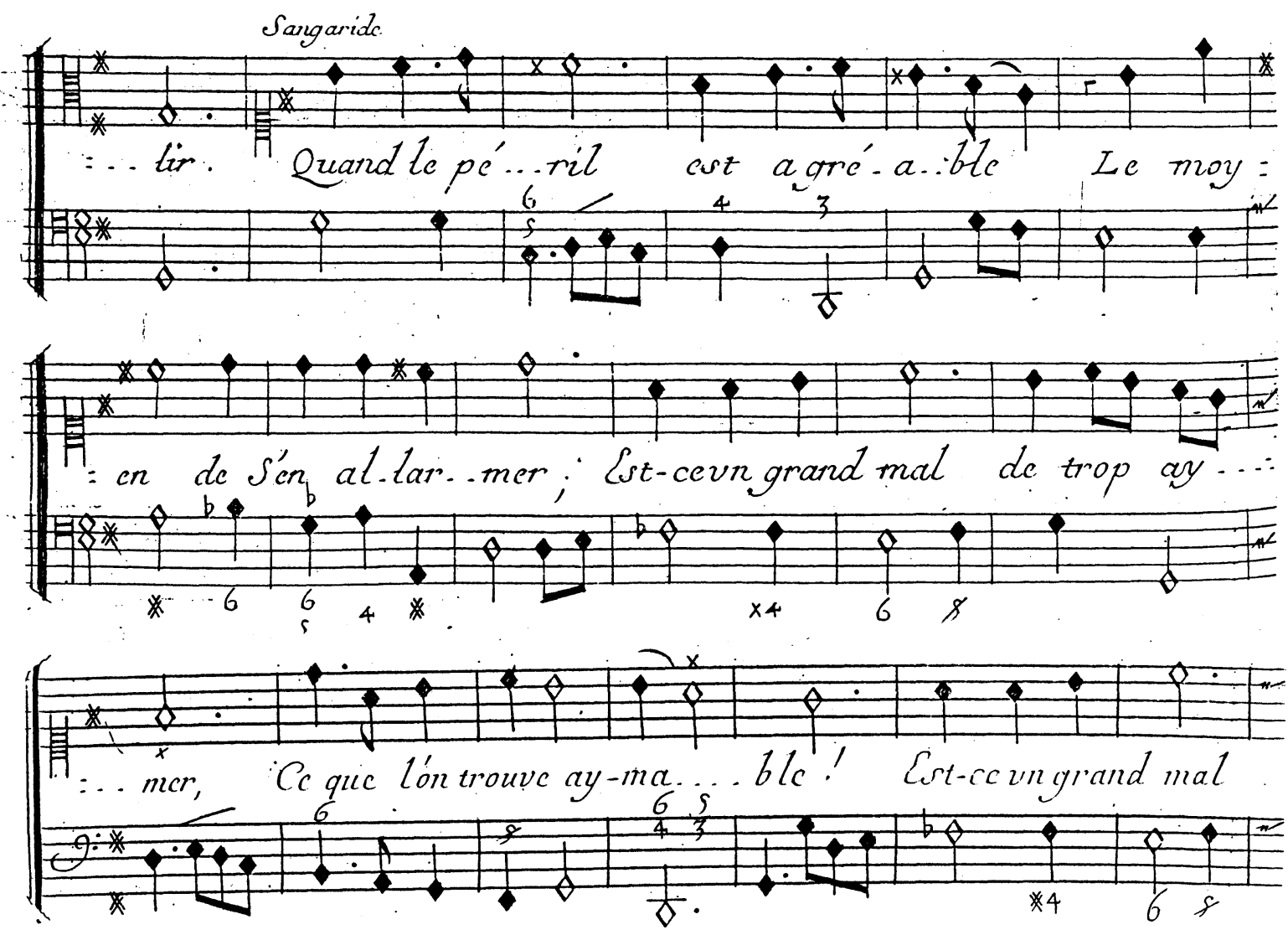

Example 2b : J.-B. LULLY, "Quand le péril est agréable”, Atys, Acte I, sc. III 


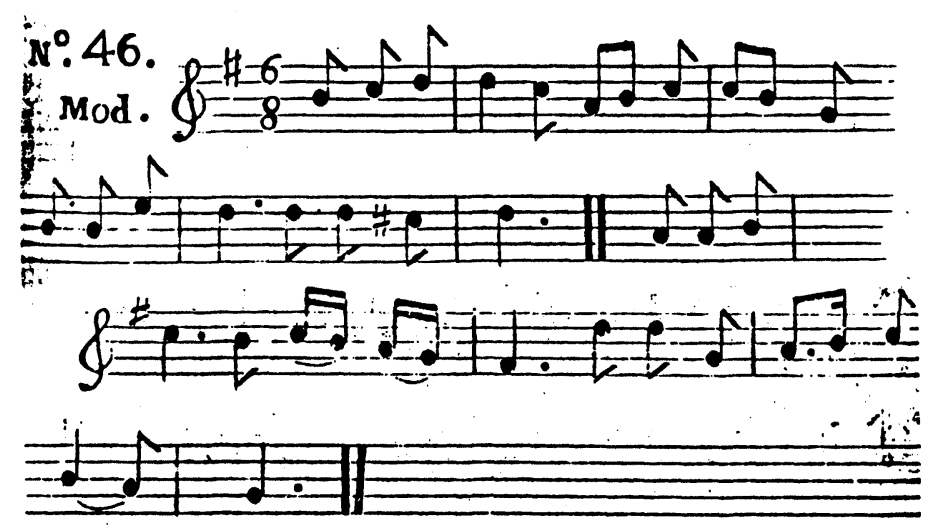

Example 2c : DAULÉ (1819), Benissez le Seigneur Suprême

Airs plus élaborés

Quelques cantiques du manuscrit sont plus élaborés que les autres et la musique en est plus complexe. Un cantique que nous n'avons pas réussi à identifier attire l'attention, Charitable Marie ( $n^{\circ} 39$ - exemple 4). Il est écrit tout au long et le refrain, chanté trois fois, entoure deux couplets. Une longue vocalise se déroule sur les syllabes « table » du premier mot du refrain, « charitable ». On trouve une vocalise semblable dans un des couplets ; les ornements et les petits phrasés sont nombreux. Comme pour certaines œuvres des Opuscules sacrés et lyriques, auxquelles il fait un peu penser, la musique a sans doute été composée spécialement pour ce cantique. S'agirait-il d'une composition de Jean Girard? Sait-on jamais.

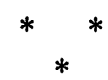

Bien qu'il existe d'autres manuscrits contenant des cantiques, le manuscrit découvert à Montréal est, à notre connaissance, le plus considérable et le seul qui contienne une telle quantité d'airs notés. La notation musicale à la manière du plain-chant en est un autre aspect particulier. Les cantiques que renferme ce livre sont diversifiés : certains étaient destinés aux catéchismes et aux missions tandis que d'autres pouvaient servir à la récréation des membres des communautés religieuses et aux personnes pieuses. Plus de la moitié d'entre eux ayant été retrouvés dans des recueils publiés en France au XVIII siècle, le manuscrit reflète bien la pratique de cette époque. Comme nombre de ces recueils ont été conservés au Québec et qu'en outre on retrouve plusieurs de ces mêmes 
Ms. no 41:

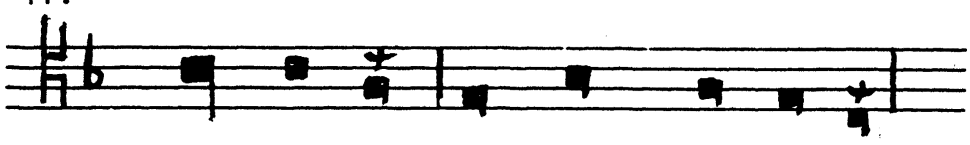

Pellegrin:

Clé du Caveau no 1000:


Daulé:

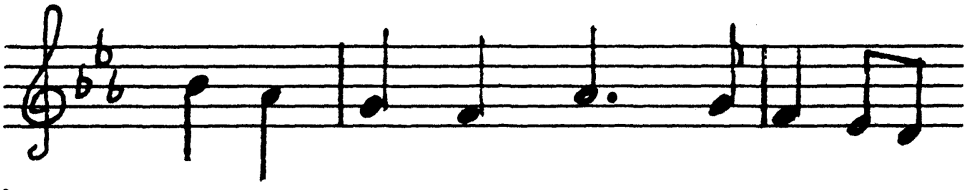

Gagnon:

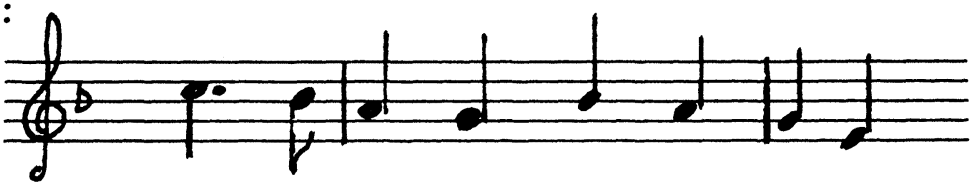

Example 3 : Cher en-fant qui de naître

cantiques dans des recueils imprimés au Québec à la fin du XVIIIe siècle et au début du XIXe ${ }^{\mathrm{e}}$, il est évident que ces cantiques sont entrés dans le patrimoine musical de la Nouvelle-France, au point que des ethnologues en ont recueilli jusqu'au $\mathrm{XX}^{\mathrm{e}}$ siècle.

Le cantique passe pour être un genre mineur, particulièrement du point de vue de la musique; en effet, c'est le texte qui compte et le timbre n'en est que le support, le véhicule. Néanmoins, un grand nombre de cantiques étaient faits sur des airs pré-existants. On y trouve des extraits du grand répertoire, des airs d'opéras qu'affectionnait Louis XIV ; d'autres timbres, les vaudevilles, étaient nés dans la rue. Ces airs, quelle que soit leur origine, ont un point en commun, celui d'être connus du public. Pour cette raison, et encore plus dans le cas de la Nouvelle-France, dont la vie musicale est encore mal connue, ce corpus de 


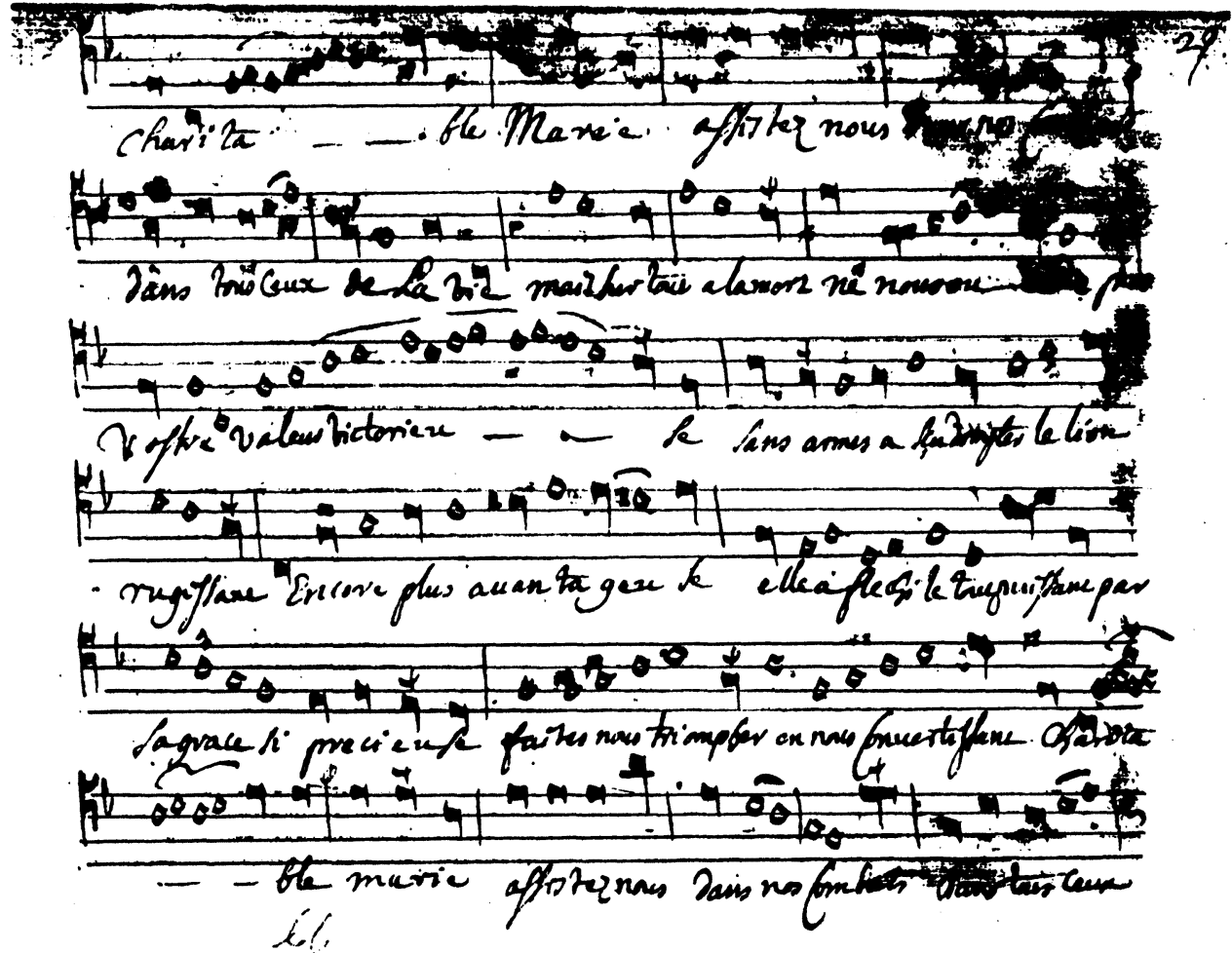

Example 4 : Cantique $n^{\circ}$ 39, Charitable Marie

cantiques, avec les airs qui lui servent de support, donne des indications fort importantes sur la musique qui s'était enracinée dans divers milieux. On apprend, en outre, quels étaient les compositeurs dont la célébrité avait franchi les mers.

Il ne faut pas oublier non plus la valeur pédagogique de cette musique. Pour de nombreuses personnes, les jeunes en particulier, le chant des cantiques représentait sans doute le seul contact avec la pratique musicale, dans une société où l'enseignement de la musique n'était pas très répandu. Il ne faut surtout pas sous-

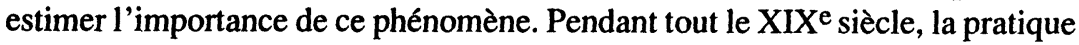
de chanter des cantiques s'est maintenue au Québec, se perpétuant jusqu'au milieu du $\mathrm{XX}^{\mathrm{e}}$ siècle. Combien de générations de jeunes se sont développé l'oreille en préparant les cantiques devant être chantés lors du prochain Salut du 


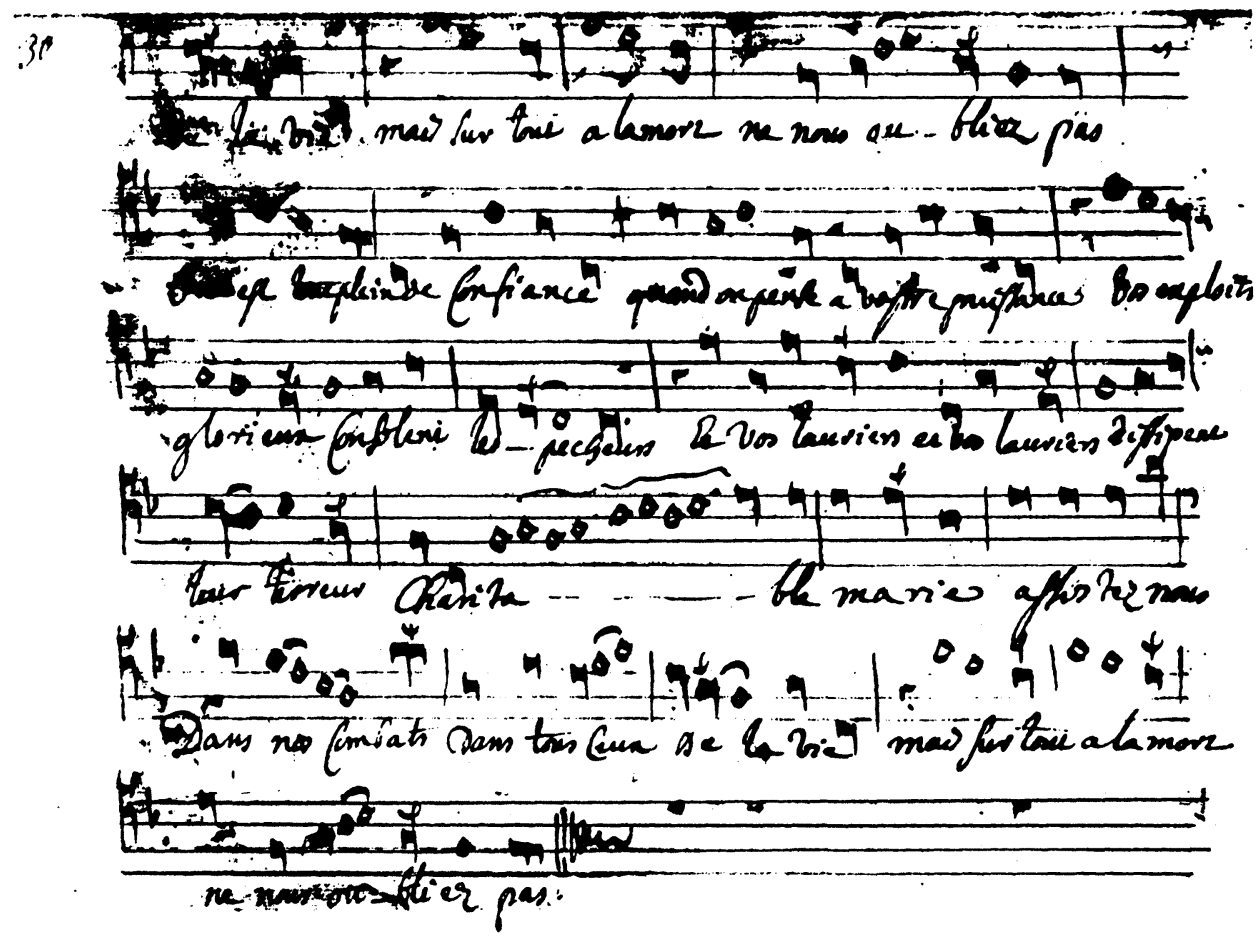

Example 4

Saint-Sacrement ? Même pour ceux qui ont pu bénéficier d'une véritable formation musicale, la pratique régulière du chant leur a permis d'intérioriser la musique et de l'intégrer tout naturellement à leur vie courante. Il est devenu de bon ton de dénigrer ce genre musical et littéraire mineur. Pourtant, depuis que dans les écoles du Québec on n'assiste plus chaque semaine au Salut, ni aux Vêpres dans les paroisses, on peut constater qu'il y a des jeunes qui ne chantent plus!

À Montréal, au XVIII ${ }^{\mathrm{e}}$ siècle, peut-être sous la conduite du Sulpicien Jean Girard, les religieux du séminaire, les élèves de la petite école des garçons, les demoiselles de la ville lors du Jubilé, avaient l'occasion, tout en louant le Seigneur, de chanter les airs connus de leur temps et, parfois, de la grande musique. 


\section{Table 4}

\section{LIVRES DES COLLECTIONS QUÉBÉCOISES DANS LESQUELS SE TROUVENT DES CANTIQUES DU MANUSCRIT DE MONTRÉAL}

1. Cantiques spirituels de l'Amour Divin, pour l'instruction \& la consolation des Ames devotes, composez par le R.P. SURIN, de la Compagnie de Jesus, Quatrième edition Revüe, corrigée \& augmentée de plusieurs beaux Cantiques, choisis dans divers auteurs bien approuvez, propres pour élever, entretenir \& unirl'Ame avec Dieu, appropriez aux trois Vies, Purgative, Illuminative \& Unitive : Et à la loüange des Saints nouvellement Canonisez

Paris, Robert Pepie, 1694

Cantique $\mathrm{n}^{0} 18$.

Archives du Monastère de l'Hôtel-Dieu de Québec Inscriptions manuscrites :

"Pour la Rde mere de St Charles Relligieuse hospitaliere » (biffée)

«Ce livre est donné a l'infirmerie par ma Sr Ste Catherine avec la permission de la Ste obeissance qui deffent de les preter » (Ces archives possèdent aussi l'édition de 1664).

2a. Cantiques spirituels sur les points les plus importants de la religion et de la morale chrétienne, accompagnez d'Hymnes pour les principales Fêtes de l'année, et à l'honneur de tous les Saints (...)

par Monsieur l'Abbé PELLEGRIN

Seconde édition, revüe, corrigée, et augmentée des cantiques sur les quinze mystères du Rosaire (...)

Paris, Nicolas Le Clerc, 1706

À la fin du volume : Airs notez des cantiques (...) Noëls Nouveaux et chansons spirituelles (1706)

Cantiques nos 36, 38, 40, 49, 50, 51.

Archives du Monastère de l'Hôtel-Dieu, Québec

Relié avec : PELLEGRIN, Chansons spirituelles (1708), Premier recueil (1709), Noëls Nouveaux (1709), Noëls Nouveaux (1711).

2b. Cantiques spirituels (...), Nouvelle édition par Monsieur l'Abbé PELLEGRIN

Paris, 1728

provenance inconnue; ex-libris gratté (l'Abbé Verreau ?)

Cantique $n^{0} 28$. 
Bibliothèque nationale du Québec, Montréal relié avec: PELLEGRIN, Chansons spirituelles (1735).

Les Archives de la Congrégation Notre-Dame, Maison généralice, Montréal, possèdent de PELLEGRIN, Cantiques spirituels (1706),

inscription manuscrite : «f.c. de Chaumaux ptre » [p.s.s.]

relié avec : PELLEGRIN, Chansons spirituelles (1708), Noëls Nouveaux, chansons et cantiques spirituels (1711), Chants des cantiques (1705),

Chants des Noëls anciens.

3. Cantiques spirituels choisis sur les points les plus importants de la Religion et de la Morale chrétienne ;

tous corrigez de nouveau, et mis sur les Airs les plus beaux et les plus connus par M.A.V.***P.M.

Angers, Veuve Jean HUBAULT, 1737

2 tomes. [Sans airs notés.]

Cantiques $n^{\text {os }} 2,3,5,6,7,10,11,19,24,32,34,35,38,46,48,49,51$.

Archives du Monastère de l'Hôtel-Dieu, Québec inscription manuscrite (tome2): «a lusage de SrSt Gabriel de lhotel Dieu ».

4. Nouveaux Cantiques Spirituels, avec des Parodies, sur les grands airs, \& les airs de Musique Instrumentale.

Paris, Jean-Baptiste GARNIER, 1750.

2 tomes.

Cantiques nos $3,5,7,8,11,33,35,40$.

Archives du Monastère de l'Hôtel-Dieu, Québec.

Après le régime français:

5. Opuscules sacrés et lyriques ou cantiques sur différents sujets de piété avec les airs notés à l' usage de la jeunesse de la paroisse de St-Sulpice

Paris, Nicolas CRAPART, 1772

2 tomes.

Cantiques $\mathrm{n}^{\text {os }} 8,11,28,35$.

Bibliothèque du Séminaire de Québec, fonds ancien. inscription manuscrite : « Lahaille».

La Bibliothèque nationale du Québec, Montréal, possède le premier tome seulement. inscription manuscrite : " Ce livre est à Monsieur Le Saulnier le curé de Montréal ». 
6. [Jean-Baptiste BOUCHER-BELLEVILLE], Recueil de cantiques à l' usage des Missions, retraites et catéchismes, $8^{e}$ édition

Québec, John Neilson, 1820 [ $1^{\mathrm{e}}$ édition, 1795] [sans airs notés]

Cantiques $\mathrm{n}^{\mathrm{os}} 2,3,6,7,8,10,11,14,28,30,34,35,40,44$.

Archives de l'Archevêché de Montréal, imprimés canadiens, et autres bibliothèques.

7. [Jean-Denis DAULÉ], Nouveau recueil de cantiques, à l' usage du diocese de Quebec, avec tous les airs notés en musique dans le meilleur gout moderne.

On y a ajouté une courte méthode pour apprendre à les mettre en plein chant. Edition qui renferme ce qu'on a trouvé de plus interessant dans les recueils anciens et modernes; sur les plus importants sujets de la religion, et les principales fetes de l'année.

Québec, Nouvelle Imprimerie, 1819.

Cantiques $\mathrm{n}^{\mathrm{os}} 2,3,6,7,8,10,11,14,19,28,30,34,35,44,49$.

Bibliothèque nationale du Québec, Montréal, et autres archives

et bibliothèques (au moins vingt exemplaires connus).

8. Cantiques de Sainte Geneviève

Paris, Delalau, 1827.

Cantiques nos 2, 3, 8, 35, 49 .

Bibliothèque du Séminaire de Québec, fonds ancien.

9. Recueil de cantiques spirituels

Avignon, 1833

Airs notés, 1861.

Cantiques nos 2, 8, 13, 28, 35, 49, 50.

Bibliothèque du Séminaire de Québec, fonds ancien.

\section{RÉFÉRENCES}

CHEYRONNAUD, J.

1988 : «Du cantique et de sa futilité »,Fiesta y Liturgica. Madrid :Casade Velázquez/ Editorial Universidad Complutense, 227-247.

FRADET, R.P.

1929: Les auvres du Bx de Montfort, poète mystique et populaire. Paris: Beauchesne.

GALLAT-MORIN, É.

1988: Un manuscrit de musique française classique - Étude critique et his- 
torique - Le Livre d'orgue de Montréal. Paris : Aux Amateurs de Livres/ Montréal : Les Presses de l'Université de Montréal.

\section{GASTOUÉ, A.}

1924 : Le cantique populaire en France. Lyon : Janin.

\section{GOSSELIN, A.}

1911 : L'instruction au Canada sous le régime français (1635-1760). Québec : Laflamme et Proulx.

HAMELINE, J.-Y.

1981 : «Le cantique sur vaudeville à l'époque de Montfort et de Pellegrin ", Ethnologie française, XI/3, 251-256.

\section{LAFORTE, C.}

1983 : Le Catalogue de la chanson folklorique française, VI : Chansons sur des timbres. Québec: Les Presses de l'Université Laval (Les Archives de folklore, 23).

[LASALLE, J.-B. de]

1705 : Cantiques qui se doivent chanter avant le Catéchisme chaque jour de la semaine ; Cantiques spirituels dans lesquels on enseigne aux Chrétiens ce qu'ils doivent croire et faire pour être sauvés; Cantiques spirituels pour les principales Fêtes et solennitez de l'année. Paris, A. Chrétien, reproduit dans les Cahiers lasalliens, $\mathrm{n}^{\circ} 22$.

1720 : Conduite des écoles chrétiennes. Avignon : J.-C. Chastanier, re-produit, avec le manuscrit de c.1704, dans les Cahiers lasalliens, $\mathrm{n}^{\circ} 24$. 